\title{
Evozierte Potenziale
}

\section{Evoked Potentials}

\author{
Autoren \\ Helmut Buchner ${ }^{1}$, Florian Klinker ${ }^{2}$, Erwin Kunesch ${ }^{3}$, Volker Milnik ${ }^{4}$, David Weise ${ }^{5}$
}

Institute

1 Klinikum Vest Recklinghausen

2 Klinik für Klinische Neurophysiologie Georg-AugustUniversität Göttingen

3 Klinik für Neurologie Bezirkskrankenhaus Mainkofen Deggendorf

4 Klinik für Neurologie St. Augustinus Krankenhaus Düren

5 Klinik für Neurologie, Schmerztherapie und Schlafmedizin Asklepios Fachklinikum Stadtroda

\section{Schlüsselwörter}

Evozierte Potenziale, Indikationen, Leitungszeit, Multiple Sklerose

\section{Key words}

Evoked potentials, Indications, Conduction time, Multiple sclerosis

\section{Bibliografie}

DOI https://doi.org/10.1055/a-0959-4626

Online-Publikation: 9.8.2019

Klin Neurophysiol 2019; 50: 149-155

(c) Georg Thieme Verlag KG Stuttgart - New York

ISSN 1434-0275

Korrespondenzadresse

Prof. Dr. med. Helmut Buchner

Klinikum Vest

Dorstener Straße 151

45657 Recklinghausen

helmut.buchner@klinikum-vest.de

\section{ZUSAMMENFASSUNG}

Evozierte Potenziale (EP; akustisch AEP, somatosensorisch SEP, visuell VEP) und die magnetisch evozierten motorischen Potenziale (MEP) liefern präzise Messungen der Leitungszeit in der stimulierten Leitungsbahn. Die Indikation zur Untersuchung der Funktionen zentraler Leitungsbahnen und peripherer Nerven ist der Nachweis oder Ausschluss einer verminderten Leitgeschwindigkeit. Der diagnostische Wert der EP liegt im Beleg für einen demyelinisierenden Typ einer Läsion bzw. Funktionsstörung. Die Indikationen für die Untersuchungen mit evozierten Potenzialen werden ohne Anspruch auf Vollständigkeit zusammengefasst. Die große Bedeutung der EP in der Diagnose und Prognose der Multiplen Sklerose wird begründet.

\section{ABSTRACT}

Evoked potentials (EP, acoustic AEP, somatosensory SEP, visual VEP) and magnetically evoked motor potentials (MEPs) provide accurate measurements of conduction time in the stimulated pathway. The indication for investigating the functions of central pathways and peripheral nerves is the demonstration or exclusion of reduced conduction velocity. The diagnostic value of an EP test lies in its ability to provide evidence for a demyelinating type of lesion or dysfunction. In this study, indications for the use of EP tests are summed up without claims to completeness. In the diagnosis and prognosis of multiple sclerosis, EP tests have an important role to play.

\author{
ABKÜRZUNGEN \\ AEP akustisch evozierte Potenziale \\ EP evozierte Potenziale \\ MEP magnetisch evozierte motorische Potenziale \\ MS Multiple Sklerose \\ SEP somatosensorisch evozierte Potenziale \\ TMS transkranielle motorische Stimulation \\ VEP visuell evozierte Potenziale \\ ZML zentrale motorische Leitungszeit
}

\section{Einleitung}

Evozierte Potenziale (EP) sind elektrische Signale, die im peripheren und zentralen Nervensystem zeitgebunden auf einen peripher applizierten artifiziellen sensorischen Reiz entstehen. Mit dem Mitteln multipler Zeitabschnitte nach dem Reiz werden zeitlich zufallsverteilte Signale, das Rauschen, minimiert und die EP extrahiert. Die Neurophysiologie und Physik der Entstehung ist gut bekannt. EPs liefern präzise Messungen der Leitungszeit im untersuchten System. Die Amplitude evozierter Potenziale sind ein grobes und wenig verlässiges Maß für die Anzahl elektrisch aktiver Nervenfasern.

Die magnetisch evozierten motorischen Potenziale (MEP) sind Summenaktionspotenziale skelettaler Muskeln, ausgelöst durch 
einzelne Entladungen eines Kondensators über eine Magnetspule. Die Neurophysiologie der Entstehung der Impulsleitung in der Pyramidenbahn ist gut bekannt. Die MEP liefern präzise Messungen der Leitungszeit in der zerebralen und spinalen motorischen Bahn und den peripheren Nerven. Die Amplitude der Muskelaktionspotenziale gibt keine zuverlässige Information über den Funktionszustand des motorischen Systems.

Der Artikel will die Indikationen der Untersuchung mit evozierten Potenzialen ohne Anspruch auf Vollständigkeit zusammenfassen. Der Einsatz der EP und MEP hat sich seit ihrer Einführung in die klinische Diagnostik deutlich verändert. Mit kritischer Betrachtung des diagnostischen Werts und der Zuverlässigkeit von Befunden, haben Indikationen an Bedeutung verloren, andere sind im diagnostischen Vorgehen etabliert.

Zur Methodik der Untersuchungen wird auf vorhandene Publikationen verwiesen [1,2].

Die Indikation zur Untersuchung der Funktionen zentraler Leitungsbahnen und peripherer Nervenmit den evozierten Potenzialen ist der Nachweis oder Ausschluss einer verminderten Leitgeschwindigkeit.

Die allgemeine Frage nach einer verminderten Leitgeschwindigkeit sollte weiter präzisiert werden in ( $\triangleright$ Abb. 1 ):

- Objektivierung von Symptomen

- Vielfach spiegeln die neurophysiologischen Befunde ein klinisches Symptom und können somit eine Läsion bestätigen.

- Lokalisation von Läsionen

- Es kann eine Läsion in der untersuchten Leitungsbahn festgestellt werden und eine Eingrenzung auf den Ort entlang der Impulsleitung erfolgen.

- Nachweis klinisch stummer Läsionen

- Eine Demyelinisierung kann zu einer messbar verminderten Leitgeschwindigkeit führen ohne dass ein klinisches Symptom besteht.

Es folgt die Frage danach, welche Leitungsbahn untersucht werden soll.

- Zur Beantwortung der Frage nach der Objektivierung von Symptomen und der Lokalisation von Läsionen wird die klinisch betroffene Bahn/Funktion untersucht.

- Zur Beantwortung der Frage nach einer klinisch stummen Läsion werden klinisch nicht betroffene Bahnen/Funktionen untersucht.

Zur Untersuchung zentraler Leitungsbahnen sind folgende Evozierte Potenziale (EP) etabliert:

- Akustisch evozierte Potenziale (AEP)

- Magnetisch evozierte motorische Potenziale (MEP)

- Somatosensorisch evozierte Potenziale (SEP)

- Visuell evozierte Potenziale (VEP)

\section{Akustisch evozierte Potenziale - AEP}

\section{Definition}

Die AEP reflektieren die Impulsleitung eines Schallreizes nach der Umwandlung in Nervenaktionspotenziale im Hörorgan. Die AEP

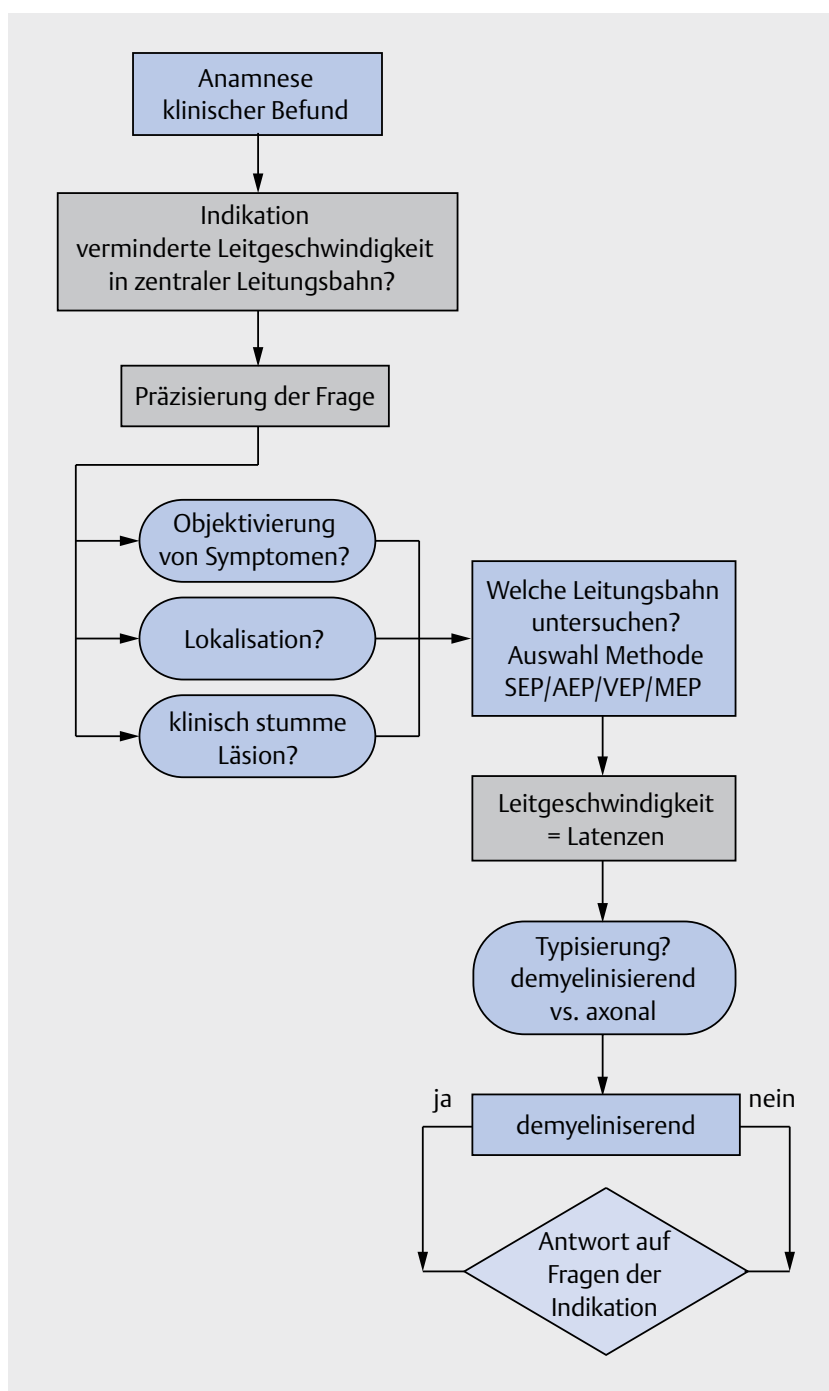

- Abb. 1 Allgemeines Vorgehen in der Diagnostik zentraler und peripherer Leitungsstörungen (Quelle: Buchner H. Zentral demyelinisierende Störungen. In: Bischoff C, Buchner H, Hrsg. SOPs Neurophysiologische Diagnostik. 1. Aufl. Stuttgart: Thieme; 2018).

entstehen im N. cochlearis und im Hirnstamm. Sie bestehen typischerweise aus 5 aufeinander folgenden positiven Potenzialgipfeln, wobei diese nach oben aufgetragen und in der Folge ihrer Latenz mit römischen Zahlen bezeichnet werden (

\section{Indikationen}

Höhergradige Schallleitungsstörungen oder cochleäre Schallempfindungsstörungen schließen eine Beurteilung der zentralen Anteile der AEP aus.

Die AEP haben in der Folge der hohen Qualität der aktuellen MRT-Technik deutlich an Bedeutung in der Diagnose von retrocochleären Hörstörungen z. B. Neurinom verloren.

In der Diagnose einer Multiplen Sklerose haben sie keinen relevanten Anteil und nur begrenzt in der Prognose, siehe Abschnitt: „Multimodale evozierte Potenziale zur Diagnose und Prognose der Multiplen Sklerose“.

Normale AEP bei komatösen Patienten sind mit einer besseren Prognose für das wieder erreichen von Wachheit verbunden. 


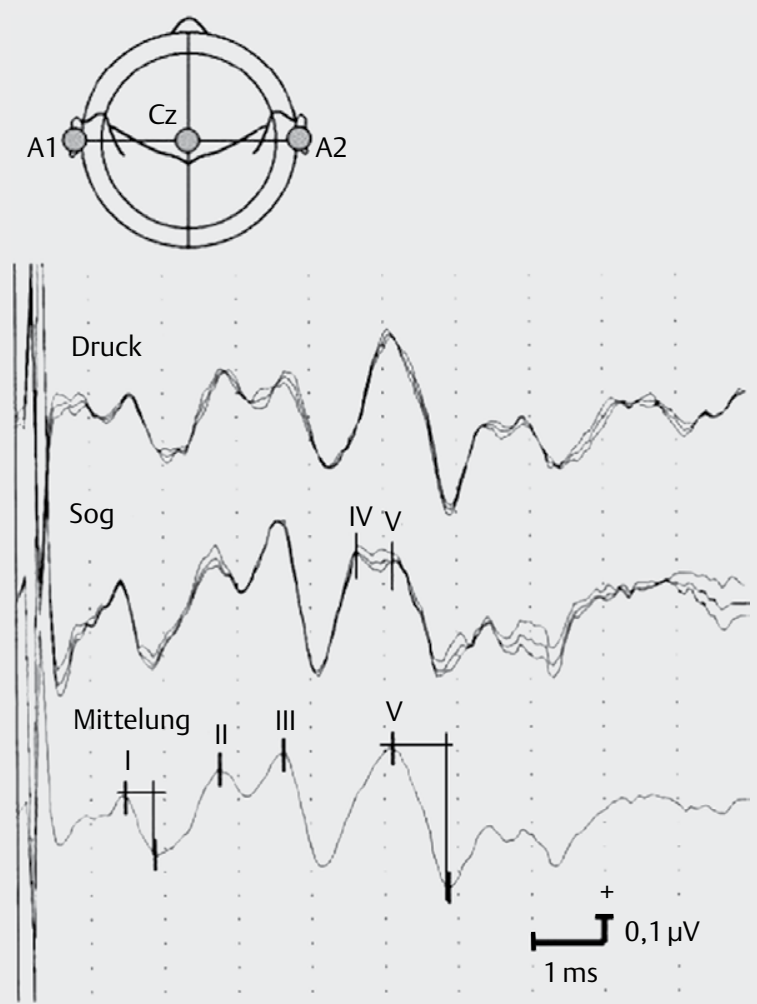

- Abb. 2 Akustisch evozierte Potenziale - Anordnung der Elektroden und typische Kurven. (Quelle: Buchner H, Claßen J, Haupt W et al. Akustisch evozierte Potenziale. In: Buchner H, Hrsg. Praxisbuch Evozierte Potenziale. 1. Auflage. Stuttgart: Thieme; 2014).

Die AEP sind als technische Zusatzuntersuchung zur Abkürzung der Wartezeit in den Kriterien zur Feststellung des irreversiblen Hirnfunktionsausfalls zugelassen [6]. Dazu ist eine Verlaufsuntersuchung mit anfangs erhaltenen Potenzialen erforderlich, was den Einsatz der Untersuchung deutlich einschränkt.

Spezielle Indikationen bestehen für das intraoperative Monitoring.

\section{Keine Indikationen}

Schwindel und Innenohrschwerhörigkeit werden klinisch bzw. mit spezifischen Tests untersucht.

\section{Kontraindikationen}

Erkrankungen des Außen- und Innenohrs mit dem Risiko einer Verschlechterung durch die Beschallung mit einem Kopfhörer. Hörgeräte und Cochleaimplantate schließen eine sinnvolle Untersuchung aus.

\section{Magnetisch evozierte motorische Potenziale - MEP}

\section{Definition}

Die MEP nach transkranieller Magnetstimulation (TMS) prüfen die Funktion zentraler motorischer Bahnsysteme. Die fraktionierte Sti- mulation kortikal und von Nervenwurzeln (oder alternativ F-Wellentechnik) gibt Auskunft über den zentralen (zentrale motorische Leitungszeit, ZML) und peripheren Schenkel des motorischen Systems ( $\triangleright$ Abb. $\mathbf{3})$.

\section{Indikationen}

Der diagnostische Nutzen besteht in der Bestimmung von Leitzeiten [7-9]. MEP haben eine klinische Bedeutung für das Therapiemonitoring sowie zur prognostischen Einschätzung bspw. nach einem Schlaganfall $[10,11]$.

Bei den folgenden aufgelisteten Erkrankungen sind MEPs diagnostisch wertvoll:

- Multiple Sklerose - Oft deutliche Verlängerung der zentralen motorischen Leitungszeit (ZML, siehe auch Kapitel Multimodale evozierte Potenziale zur Diagnose und Prognose der Multiplen Sklerose)

- Schlaganfall - Bei schwerer Hemiparese bzw. Hemiplegie keine Reizantwort nach Stimulation der betroffenen Hemisphäre; Prognose besser, wenn zentrale Leitzeit normal oder gering verlängert.

- Erkrankungen des Rückenmarks

- Bei zervikaler Myelopathie und Kompression in mehreren Segmenten kann durch Ableitung entsprechender Kennmuskeln das Segment mit der ausgeprägtesten Funktionsstörung eingegrenzt werden.

- Bei kompletter Rückenmarksschädigung gibt es nach kortikaler Stimulation keine Reizantworten unterhalb der Läsion.

- Bei VitB12-Mangel mit subakuter Degeneration führt zu einer verlängerten zentralen Leitungszeit.

- Bewegungsstörungen - zentrale Leitzeit bei vielen Erkrankungen einschließlich M. Parkinson meist normal [12].

- Amyotrophe Lateral-Sklerose (ALS) - zentrale Leitzeit kann, muss aber nicht verlängert sein; wenn sie verlängert ist, ist dies zur differentialdiagnostischen Abgrenzung gegenüber einer Multifokalen motorischen Neuropathie nützlich. Differentialdiagnostisch: zentrale Leitzeit bei zervikaler Myelopathie meist stärker verlängert als bei ALS. Ableitung von Zunge oder anderen orofazialen Muskeln kann die Affektion zentraler Bahnsysteme (kortikobulbärer Trakt) belegen [13]. Die Schwellenreizstärke ist im Frühstadium der ALS durch glutamat-induzierte kortikale Hyperexzitabilität manchmal vermindert, in späteren Stadien erhöht [14]. Die Bestimmung ist jedoch technisch schwierig. Die Amplituden bei konventionellen MEPs sind wenig aussagekräftig. Die Triple-Stimulation gibt Informationen zur Degeneration des kortikospinalen Trakts, ist aber schmerzhaft und wird daher im klinischen Alltag kaum eingesetzt [15-17].

- Primäre Lateralsklerose: Fehlende Reizantworten nach TMS (transkranieller Stimulation) oder zentrale Leitungszeit deutlich verlängert.

- Funktionelle Störungen mit „pseudo“-Paresen haben normale MEP (CAVE ausreichende Vorinnervation).

- Mögliche seltene Indikationen sind: Atrophie Typ SCA1 [18]; M. Friedreich. 


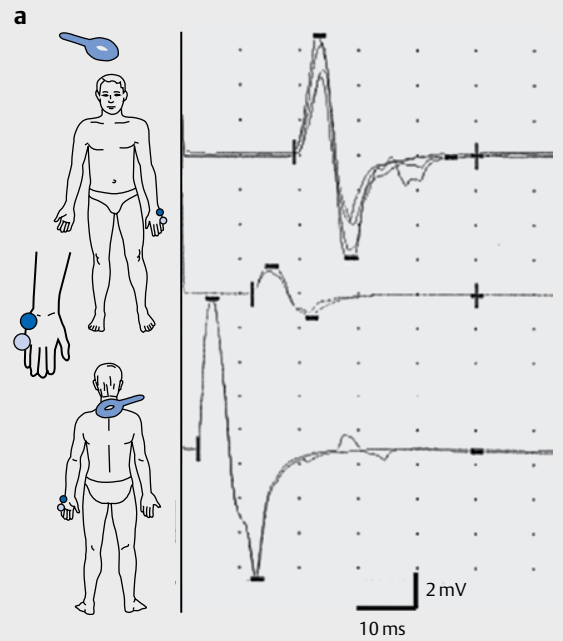

b

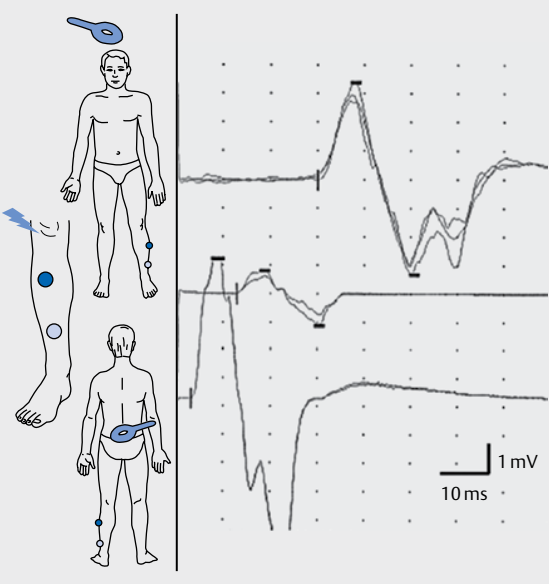

- Abb. 3 a Magnetisch evozierte motorische Potenziale zur Hand - Anordnung der Elektroden und typische Kurven. b Magnetisch evozierte motorische Potenziale zum Bein - Anordnung der Elektroden und typische Kurven. (Quelle: Buchner H, Milnik V. Parameter. In: Bischoff C, Buchner H, Hrsg. SOPs Neurophysiologische Diagnostik. 1. Auflage. Stuttgart: Thieme; 2018) [3].

\section{Kontraindikationen}

- absolute Kontraindikation ist ein metallisches Objekt/Gerät in der Nähe der Magnetspule wie z. B. Kabel einer Tiefen Hirnstimulation, Cochlearimplantat oder Medikamentenpumpen [19].

- Keine Untersuchung, wenn die evozierte Kontraktion der Muskulatur Nebeneffekte haben kann z. B. bei instabiler Fraktur der Wirbelsäule.

- Relative Kontraindikationen bestehen wenn eine der folgenden Fragen bejaht wird. Dann muss die Indikation streng gestellt werden und der Patient über ein mögliches Riskiko aufgeklärt werden (vom Autor übersetzt nach [20]):

(1) Haben sie eine Epilepsie oder hatten sie jemals einen epileptischen Anfall?

(2) Hatten sie jemals eine Ohnmacht oder einen Kreislaufzusammenbruch (Synkope)? Wenn Ja, beschreiben sie bitte das Ereignis?

(3) Hatten sie jemals eine Kopfverletzung mit einer Bewußtlosigkeit oder wurde eine Hirnkontusion diagnostiziert?

(4) Haben sie eine Hörstörung oder ein Ohrgeräusch?

(5) Haben sie ein Cochlearimplantat?

(6) Bei Frauen: Sind sie schwanger oder könnten sie es sein?

(7) Haben sie etwas Metallisches im Kopf oder an einer anderen Körperstelle (z. B. OP-Clip, Splitter etc.)? Wenn Ja, können sie genau sagen wo und welches Metall?

(8) Haben sie einen implantierten Neurostimulator (z. B. Tiefe Hirnstimulation, Spinale Stimulation, Vagus-Nerv-Stimulator)? Achtung: Siehe Absolute Kontraindikationen!

(9) Haben sie einen Herzschrittmacher oder Kabel in der Brust?

(10) Tragen sie eine Medikamentenpumpe?

(11) Nehmen sie Medikamente? Wenn Ja, welche?

(12) Hatten sie schon mal eine MEP-Untersuchung? Wenn Ja, gab es irgendein Problem?

(13) Hatten sie schon mal eine MRT (Kernspintomografie)? Wenn Ja, gab es irgendein Problem?

\section{Somatosensorisch evozierte Potenziale - SEP Definition}

Die somatosensorisch evozierten Potenziale (SEP) messen die Leitzeiten in der lemniscalen Leitungsbahn [21, 22]. In dieser werden die sensiblen Qualitäten von Mechanorezeptoren (taktile Objekterkennung, Lokalisation von Hautberührung; Detektion von Vibration und Texturen) und von Proprioceptoren (Gelenkposition, Bewegung und Kraft) geleitet. Die SEP können deshalb Leitungsstörungen der lemniscalen Bahn belegen, aber Störungen im Tractus spinothalamicus (Thermo- und Schmerzrezeption) nicht ausschließen.

Für die klinische Routine und deshalb hier darauf beschränkt, werden der N.medianus bzw. der N.tibialis mit Rechteck-Stromimpulsen stimuliert. Die afferenten Impulse werden über den peripheren Nerven zum Spinalganglion, der Hinterstrangbahn zum Lemniscus medialis und über den Thalamus zum sensorischen postzentralen Kortex geleitet.

Die SEP werden mit Elektroden platziert entlang der Leitungsbahn gemessen. Das N.medianus SEP: N9 - entsteht im Plexus brachialis; N13 - in zervikalen segmentalen Interneuronen; N14 - im Lemniscus medialis und N20 - im somatosensorischen Kortex. Das N.tibialis SEP: N22 - entsteht im lumbalen Plexus;

P40 - im somatosensorischen Kortex ( Abb. 4,5).

\section{Indikationen}

Nach den Abschnitten der Leitungsbahn gliedern sich klinische Indikationen:

Bestimmung der Leitungszeit im: peripheren Nerven, zentrale lemniscale Bahn (Rückenmark und Hirnstamm), Thalamus zum Kortex.

\section{Periphere Nerven}

In Ergänzung zur Neurografie untersuchen die SEP proximale Abschnitte peripherer Nerven. Die F-Welle gilt für die Diagnostik von Polyneuropathien mit proximalem Schwerpunkt als sensitiver und zuverlässiger. Nach hochgradigen Läsionen einzelner Nerven kön- 


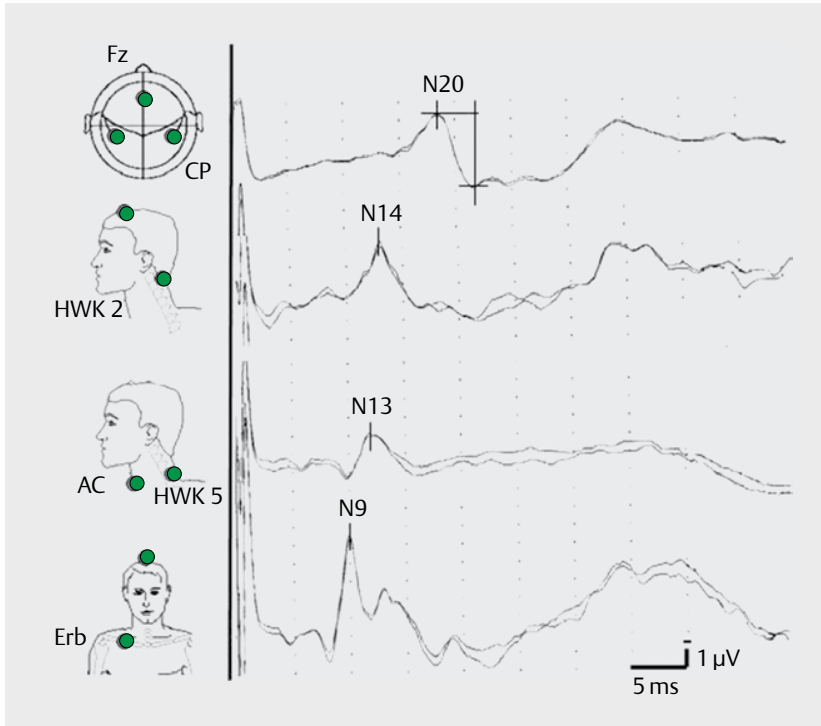

Abb. 4 Somatosensorisch evozierte Potenziale. N. medianus - Anordnung der Elektroden I und typische Kurven. (Quelle: Buchner $\mathrm{H}$, Claßen J, Haupt W et al. Somatosensorisch evozierte Potenziale. In: Buchner H, Hrsg. Praxisbuch Evozierte Potenziale. 1. Auflage. Stuttgart: Thieme; 2014)

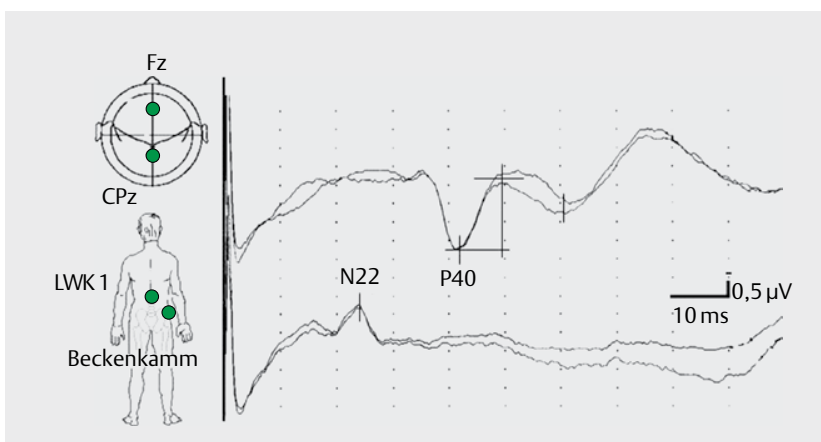

- Abb. 5 Somatosensorisch evozierte Potenziale. N. tibialis - Anordnung der Elektroden und typische Kurven. (Quelle: Buchner $\mathrm{H}$, ClaBen J, Haupt W et al. Somatosensorisch evozierte Potenziale. In: Buchner H, Hrsg. Praxisbuch Evozierte Potenziale. 1. Auflage. Stuttgart: Thieme; 2014).

nen SEP erhalten bzw. wieder messbar sein, auch wenn keine motorische oder sensible Neurografie über den Läsionsort möglich ist.

Bei Läsionen einzelner Nervenwurzeln sind die Standard N.medianus und N.tibialis-SEP wegen des polysegmentalen Ursprungs der stimulierten Nerven normal.

\section{Zentrale lemniscale Leitungsbahn}

Die SEP können eine verlängerte Leitungszeit dem spinalen, Hirnstamm oder kortikalen Abschnitt der Bahn zuordnen. Einen Schluss auf die Ursache lassen die SEP nicht zu (wie auch die anderen EPs).

Die N.medianus-SEP sind bei ca. $50 \%$ und die N.tibialis-SEP in ca. $70 \%$ aller Patienten mit gesicherter Multipler Sklerose pathologisch. Wenn keine zentralen somatosensorischen Symptome bestehen, werden dennoch in ca. 30 \% der Patienten pathologische N.tibialis-SEP gemessen.
Spinale Läsionen, traumatisch oder kompressiv, mit über mehr als eine Woche anhaltendem Ausfall der SEP, haben eine schlechte Prognose.

Bei einer vermuteten cervikalen Myelopathie belegt eine verlängerte Interpeaklatenz N13-N20 eine funktionelle relevante Störung.

Bei Läsionen nicht demyelinisierender Ursache im Hirnstamm, Thalamus oder thalamo-kortikal sind die Latenzen in der Regel normal und die Amplitude der kortikalen Potenziale normal, gemindert oder das SEP ausgefallen.

Bei Patienten im Koma, verursacht durch eine primäre oder sekundäre Hirnschädigung, ist ein bilateraler Ausfall der kortikalen N20 bei erhaltenen subkortikalen Potenzialen N14 und N13 mit einer infausten Prognose des Wiedererlangen von Wachheit verbunden, wenn der Befund erhoben wurde mehr als 24 Stunden nach Wiederherstellung der Herzkreislauffunktion und ohne Hypothermie bzw. mehr als 72 Stunden, wenn zuvor eine Behandlung mit Hypothermie erfolgte. Es wurden wenige Ausnahmen von dieser Regel berichtet, dann bestanden entweder eine Hypothermie oder bilaterale Läsionen im postzentralen Kortex. Bilateral erhaltene N20 sind ohne Vorhersagewert.

\section{Kortikaler Myoklonus}

In der Amplitude erhöhte SEP (sogenannte Riesen-SEP, N20-P25 Amplitude $>15 \mu \mathrm{V}$ ) werden bei Patienten mit einem kortikalen Myoklonus gefunden, meist bei Myoklonusepilepsie oder hypoxischen Myoklonien.

\section{Irreversibilitätsnachweis des Hirnfunktionsausfalls}

Die N.medianus-SEP sind zum Irreversibilitätsnachweis des Hirnfunktionsausfalls bei primären supratentoriellen und bei sekundären Hirnschädigungen jenseits des vollendeten 2 . Lebensjahrs geeignet [6].

\section{Intraoperatives Monitoring}

Die SEP haben etablierte Indikationen im intraoperativen Monitoring. Dazu wird verwiesen auf Cruccu et al. [23].

\section{Kontraindikationen}

Keine Stromstimulation in unmittelbarer Nähe von implantierten Kabeln und Impulsgebern, einer Tiefen Hirnstimulation oder von Herzschrittmachern/Defibrillatoren. Keine Stromstimulation in unmittelbare Nähe von Zentralvenenkathetern [24].

\section{Visuell evozierte Potenziale - VEP}

\section{Definition}

Die visuell evozierten Potentiale ermöglichen die Untersuchung der Sehbahn. Durch visuelle Reize erfolgt eine Stimulation der Netzhaut. Abgeleitet wird die elektrische Reizantwort okzipital $(\mathrm{Oz})$. Die Reizweiterleitung innerhalb der Netzhaut erfolgt zunächst potentialkodiert, was die im Vergleich zu anderen EP langen Latenzen erklärt. Ab den Ganglion-Zellen erfolgt eine Spike-kodierte Reizweiterleitung. Da das menschliche Auge auf die Darstellung von Kontrasten ausgelegt ist, lassen sich mit Schachbrettmusterumkehrreize die stabilsten VEP evozieren. Die typischen Potentialkomponenten sind hier: N70, P100 (N140). VEP durch Blitzlichtstimulation hängen weniger von der Mitarbeit des Probanden ab, weisen jedoch eine deutlich höhere inter- und intraindividuelle Variabilität 


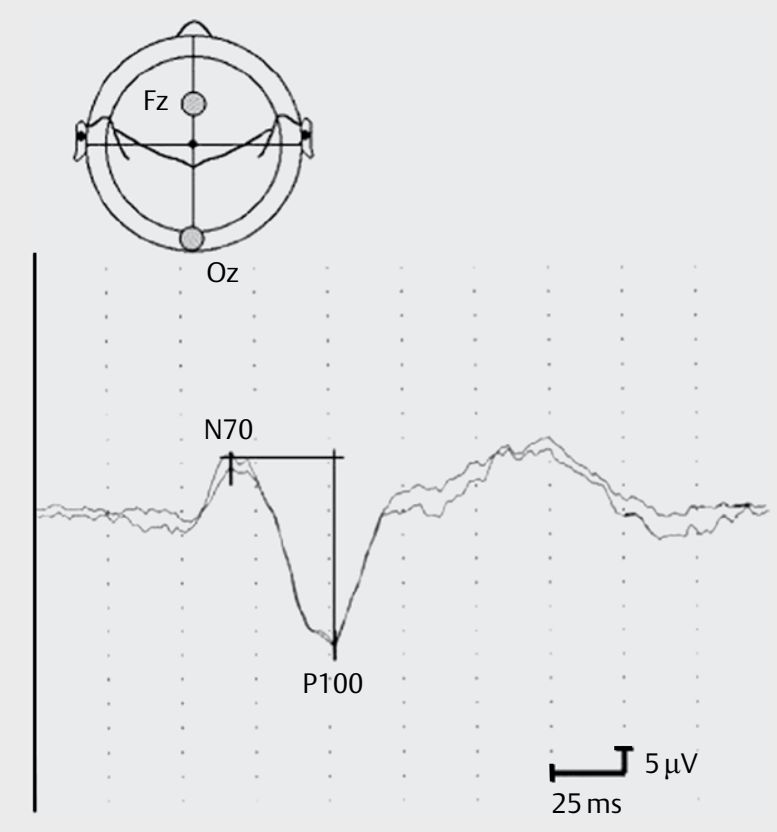

Abb. 6 Visuell-evozierte Potenziale - Anordnung der Elektroden und typische Kurven. (Quelle: Buchner $\mathrm{H}$, Claßen J, Haupt W et al. Somatosensorisch evozierte Potenziale. In: Buchner H, Hrsg. Praxisbuch Evozierte Potenziale. 1. Auflage. Stuttgart: Thieme; 2014).

auf, weshalb sie von sehr begrenztem klinischen Nutzen sind [25] (• Abb. 6).

\section{Indikation}

Aufgrund der Reorganisation der Nervenfasern im Chiasma opticum sind nur prächiasmatische Prozesse durch eine monokuläre Ganzfeldstimulation sicher feststellbar. Für die Untersuchung retrochiasmatischer Prozesse ist prinzipiell eine Halbfeldstimulation möglich. Aufgrund oft schlechterer Darstellbarkeit ist diese Methode für die klinische Routine allerdings nur begrenzt geeignet [26]. Wie alle EP identifizieren die VEP demyelinisierende Schädigungen besonders sensitiv. Folglich ist die Objektivierung einer klinisch manifesten oder der Nachweis einer klinisch inapparenten Neuritis nervi optici, z. B. bei einer MS, die wichtigste Indikation der VEP [26, 27]. Die Darstellung funktioneller Störungen durch andere Erkrankungen ist mit dem VEP möglich. Beispiele sind [25]:

- Ischämische Opticus-Neuropathie (arteriitische/nicht-arteriitische ischämische anteriore Opticus-Neuropathie)

- Kompressionen des N. opticus (z. B. Tumor, bilaterale Mehrkanal-Ableitung hilfreich)

- Hereditäre Optikopathien (z. B. Leber'sche Opticus-Atrophie)

- Stoffwechselbedingte Optikopathien (z. B. Vitamin B12-Mangel)

\section{Kontraindikation}

Kontraindikationen i.S. einer möglichen Schädigung des Probanden bestehen nicht. Es sind jedoch Einschränkungen der Aussagekraft der VEP zu berücksichtigen:

- Periphere Gesichtsfeldanteile tragen nicht in relevantem Umfang zu den VEP bei. Daher eignen sich herkömmliche VEP nicht zur Objektivierung von Gesichtsfelddefekten. Eine mögliche Alternative bieten multifokale VEP [1].

- Bei deutlicher Fehlsichtigkeit (Visus schlechter als 20/200) ist eine zuverlässige Untersuchung mit Schlachbrettmusterumkehrreizen nicht möglich [1]

- Bei fehlender Kooperation des Probanden (fehlende Fixierung, starke Müdigkeit) ist keine Untersuchung mit sicherem Befund möglich [25].

\section{Multimodale evozierte Potenziale zur Diagnose und Prognose der Multiplen Sklerose}

\section{Diagnose}

Die aktuellen diagnostischen Kriterien der Multiplen Sklerose nutzen ausschließlich die VEP als objektive technische Untersuchung zur Prüfung der Evidenz eines klinischen Befunds [28].

Die EP können jedoch (auch subjektive) klinische Symptome objektivieren und quantifizieren, klinisch stumme Läsionen identifizieren, beitragen zur Lokalisation von Läsionen und ggf. ihren demyelinisierenden Charakter sichern. Der Beitrag der EP zur Diagnose einer MS ist im Vergleich zu den aktuellen Kriterien der Magnetresonanz Tomografie nicht untersucht, können aber sensitiver hinsichtlich von Hirnstammläsionen sein als die MRT [29, 30].

Die VEP sind die etablierte Untersuchung zur Sicherung einer Neuritis N.optici. Sie sind sensitiver als die klinische Untersuchung und vermutlich auch als die optische Kohärenztomography (OCT) [31].

Die SEP und MEP sind sensitive Untersuchungen zur Prüfung der „langen“ somatosensorischen bzw. motorischen Bahnen. Sie haben einen bedeutsamen Beitrag in der Identifikation von spinalen Läsionen und der Klärung deren klinischer Relevanz.

Die AEP haben eine geringe Sensitivität auf Grund der sehr kurzen untersuchen Strecke durch den N. cochlearis und den Hirnstamm, können aber bei Verdacht auf eine Hirnstammaffektion sinnvoll sein.

Die Autoren sind der Meinung, dass zur Erst- und Differentialdiagnose sowie zum Monitoring des Krankheitsverlaufs auf die Zusatzinformation durch die EP nicht verzichtet werden sollte.

\section{Prognose}

Die Bedeutung der multimodalen EP zur Prognose des Verlaufs einer MS wurde in mehr als 8 unabhängigen Studien untersucht $[32,33]$. Dazu wurde in den meisten Studien ein Summenwert der EP zusammengefasst: 0 = normal; 1 = verlängerte Latenz, 2 = verlängerte Latenz plus veränderte Form des Potenzials, 3 = Ausfall des Potenzials. Die Punktwerte wurden für jede untersuchte Modalität und Körperseite addiert. In allen Studien war ein niedriger Summenwert mit einer günstigeren Prognose verbunden.

In einer aktuellen Untersuchung mit AEP, VEP, SEP und MEP war ein Summenwert von über 4, erhoben binnen 6 Monaten nach der Diagnose, verbunden mit einem Risiko einer Verschlechterung des EDSS von einem oder mehr Punkten von $60 \%$ binnen 10 Jahren. Dagegen ein Summenwert von 4 oder kleiner mit einem Risiko von lediglich $16 \%$ [33].

Zur Beurteilung der Schädigung der Sehbahn sollten VEPs nicht im akuten Schub einer Optikusneuritis sondern im Intervall untersucht werden [34]. 
Die multimodalen EP geben ein objektives Kriterium zur Einschätzung der Prognose einer kürzlich diagnostizierten MS und könnten somit einen Beitrag hinsichtlich Therapieentscheidung beitragen [35].

\section{Interessenkonflikt}

Die Autoren geben an, dass kein Interessenkonflikt besteht.

\section{Literatur}

[1] Buchner H., (Hrsg.). Praxisbuch - Evozierte Potenziale. Stuttgart: Thieme; 2014

[2] Buchner H. Evoked Potentials. in Mills KR, (Hrsg.). Clinical Neurophysiology Oxford. University Press; 2016: 175-186

[3] Bischoff Ch, Buchner H., (Hrsg.). SOPs Neurophysiologische Diagnostik. Stuttgart: Thieme; 2018

[4] Pratt $\mathrm{H}$, Aminoff M, Nuwer MR, et al. Short-latency auditory evoked potentials.In Deuschl G, Eisen A., (Hrsg.). Recommendations for the practice of clinical neurophysiology. Elektroenceph clin Neurophysiol Amsterdam.Elsevier; 1999: (Suppl 52): 69-77

[5] Legatt AD. Brainstem auditory evoked potentials: methodology, interpretation, and clinical application. In Aminoff MJ, (Hrsg.). Aminoff's electrodiagnosis in clinical neurology. 6. Aufl. Amsterdam: Elsevier; 2012: 519-552

[6] Wissenschaftlicher Beirat der Bundesärztekammer. Richtlinie gemäß § 16 Abs. 1 S. 1 Nr. 1 TPG für die Regeln zur Feststellung des Todes na $h$ $\S 3$ Abs. 1 S. 1 Nr. 2 TPG und die Verfahrensregeln zur Feststellung des endgültigen, nicht behebbaren Ausfalls der Gesamtfunktion des Großhirns, des Kleinhirns und des Hirnstamms nach § 3 Abs. 2 Nr. 2 TPG. 4. Fortschreibung. Dtsch Ärztebl 2015; 112: A-1256

[7] Chen R, Cros D, Curra A. The clinical utility of transcranial magnetic stimulation: Report of an IFCN committee Clin Neurophysiol 2008; 119: 504-532

[8] Groppa S, Oliviero A, Eisen A et al. A practical guide to diagnostic transcranial magnetic stimulation: Report of an IFCN committee. Clin Neurophysiol 2012; 123: 858-882

[9] Mills KR. Clinical Neurophysiology (Chapter14: Transcranial magnetic stimulation). 163-173 Oxford Univ. Press; 2017

[10] Ayache SS, Creange A, Farhat WH et al. Relapses in multiple sclerosis: effects of high-dose steroids on cortical excitability. Europ J of Neurology 2014; 21: 630-636

[11] Trompetto C, Assini A, Buccolieri A, Marchese R, Abbruzzese G. Motor recovery following stroke: a transcranial magnetic stimulation study. Clin. Neurophysiol 2000; 111: 1860-1867

[12] Cantello R. Applications of transcranial magnetic stimulation in movement disorders. J of Clin Neurophysiology 2002; 19: 272-293

[13] Urban PP, Vogt T, Hopf HC. Corticobulbar tract involvement in amyotrophic lateral sclerosis. A transcranial magnetic stimulation study. Brain 1998; 121: 1099-1108

[14] Vucic S, Ziemann U, Eisen A, Hallett M, Kiernan MC. Transcranial magnetic stimulation and amyotrophic lateral sclerosis: pathophysiological insights. J Neurol Neurosurg Psych 2013; 84: 1161-1170

[15] Magistris MR, Rösler KM, Truffert A, Landis T, Hess CW. A clinical study of motor evoked potentials using a triple stimulation technique. Brain 1999; 122: 2208-2218

[16] Rösler KM, Truffert A, Hess CW, Magistris MR. Quantification of upper motor neuron loss in amyotrophic lateral sclerosis. Clin Neurophysiol 2000; $111: 2208-2218$
[17] Attarian S, Verschueren A, Pouget J. Magnetic stimulation including the triple-stimulation technique in amyotrophic lateral sclerosis. Muscle \& Nerve 2007; 36: 55-61

[18] Schwenkreis P, Tegenthoff MWitscher et al. Motor cortex activation by transcranial magnetic stimulation in ataxia patients depends on the genetic defect. Brain 2002; 125: 301-309

[19] Rossi S, Hallet M, Rossini PM et al. Safety, ethical considerations, and application guidelines for the use of transcranial magnetic stimulation in clinical practice and research. Clin Neurophysiol 2009; 120: 2008-2039

[20] Rossi S, Hallett M, Rossini PM et al. Screening questionnaire before TMS: An up date. Clin Neurophysiol 2011; 122: 1686

[21] Mauguière $F$, Allison T, Babiloni $C$ et al. Somatosensory evoked potentials. In Deuschl G, Eisen A, Hrsg. Recommendations for the practice of clinical neurophysiology. Elektroenceph clin Neurophysiol Amsterdam. Elsevier; 1999: (Suppl 52): 79-90

[22] Kimura J. Electrodiagnosis in diseases of nerve and muscle - principles and practice. 4. Aufl. Oxford: Oxford University Press; 2013

[23] Cruccu G, Aminoff M], Curio G et al. Recommendations for the clinical use of somatosensory- evoked potentials. Clinical Neurophysiology 2008; 119: 1705-1719

[24] Hinrichs H. Sicherheit der Stromstimulation für implantierte elektrische Stimulatoren - Stellungnahme der technischen Kommission der DGKN Klin Neurophysiol 2017; 47: 1-5

[25] Robson AG, Nilsson J, Jalali $S$ et al. ISCEV guide to visual electrodiagnostic procedures. Doc Ophthalmol 2018; 136: 1-26

[26] Celesia GG, Brigell M, Recommended standards for pattern electroretinograms and visual evoked potentials. In: Deuschl G, Eisen A., Hrsg. Recommendations for the practice of clinical neurophysiology. Elektroenceph clin Neurophysiol Amsterdam.Elsevier; 1999: (Suppl 52): 53-67

[27] Holder GE, Celesia GG, Miyake Y et al. International federation of Clinical neurophysiology: recommendations for visual system testing. Clinical Neurophysiology 2010; 121: 1393-1409

[28] Thompson AJ, Banwell BL, Barkhof $F$ et al. Diagnosis of multiple sclerosis: 2017 revisions oft he McDonald criteria Lancet Neurol. 2018; 17: 162-173

[29] Magnano I, Pes GM, Pilurzi G et al. Exploring brainstem function in multiple sclerosis by combining brainstem reflexes, evoked potentials, clinical and MRI investigations Clin Neurophysiol 2014; 125: 2286-2296

[30] Magnano I, Pes GM, Cabboi MP et al. Comparison of brainstem reflex recordings and evoked potentials with clinical and MRI data to assess brainstem dysfunction in multiple sclerosis: a short-term follow-up Neurol Sci 2016; 37: 1457-1465

[31] Behbehani R, Ahmed S, Al-Hashel J et al. Sensitivity of visual evoked potentials and spectral domain optical coherence tomography in early relapsing remitting multiple sclerosis Mult Scler Relat Disord 2017; 12: $15-19$

[32] Buchner H. Der „alte“ und der „neue“ Stellenwert der Evozierten Potenziale in der Diagnose und Prognose der Multiplen Sklerose Klein Neurophysiol 2013; 44: 1-6

[33] London F, El Sankari S, van Pesch V. Early disturbances in multimodal evoked potentials as a prognostic factor for long-term disability in relapsing-remitting multiple sclerosis patients Clin Neurophysiol 2017; 128: 561-569

[34] Schlaeger R, D'Souza M, Schindler C et al. Prediction of MS disability by multimodal evoked potentials: investigation during relapse or in the relapse-free interval? Clin Neurophysiol 2014; 125: 1889-1892

[35] Hardmeier M, Leocani L, Fuhr P. A new role for evoked potentials in MS? Repurposing evoked potentials as biomarkers for clinical trials in MS Mult Scler 2017; 10: 1309-1319 